\title{
The Effect of Conductivity Values on ST Segment Shift in Subendocardial Ischaemia
}

\author{
Peter R. Johnston* and David Kilpatrick
}

\begin{abstract}
The aim of this study was to investigate the effect of different conductivity values on epicardial surface potential distributions on a slab of cardiac tissue. The study was motivated by the large variation in published bidomain conductivity parameters available in the literature.

Simulations presented are based on a previously published bidomain model and solution technique which includes fiber rotation. Three sets of conductivity parameters are considered and an alternative set of nondimensional parameters relating the tissue conductivities to blood conductivity is introduced. These nondimensional parameters are then used to study the relative effect of blood conductivity on the epicardial potential distributions.

Each set of conductivity parameters gives rise to a distinct set of epicardial potential distributions, both in terms of morphology and magnitude. Unfortunately, the differences between the potential distributions cannot be explained by simple combinations of the conductivity values or the resulting dimensionless parameters.
\end{abstract}

Index Terms-Anisotropy, bidomain model, conductivity values, simulation, ST depression, subendocardial ischemia.

\section{INTRODUCTION}

D EPRESSION of the ST segment of the electrocardiogram has been recognized as a sign of ischemia for many years [1]. However, most explanations of the mechanisms responsible for this depression have been controversial [2]. In an attempt to further our understanding of this phenomenon, we recently introduced a mathematical model for ST segment changes due to subendocardial ischemia in a slab of cardiac tissue [3]. The model was based on the bidomain representation of cardiac tissue [4] and the tissue conductivity values of Clerc [5] were used to perform the simulations.

The aim of our previous study was to investigate the spatial change in epicardial potentials as the degree of subendocardial ischemia increased to full thickness. We also considered the effect of simplifying assumptions such as isotropic cardiac tissue and ignoring fiber rotation.

The aim of this present study is to investigate the effect the actual conductivity values have on the epicardial surface potential distribution on the slab of cardiac tissue. Here, we are interested in the spatial distribution of ST segment change, not the temporal and, thus, the source chosen is static and represen-

Manuscript received March 15, 2002; revised September 16, 2002. This work was supported by the National Health and Medical Research Council of Australia and the National Heart Foundation of Australia. Asterisk indicates corresponding author.

*P. R. Johnston is with the School of Science, Griffith University, Nathan, Queensland, Australia, 4111 (e-mail: P.Johnston@mailbox.gu.edu.au).

D. Kilpatrick is with the Department of Medicine, University of Tasmania, GPO Box 252-34, Hobart, Tasmania, 7001, Australia.

Digital Object Identifier 10.1109/TBME.2002.807660 tative of a typical source in the center of the ST interval. This study was motivated by the wide variability in published bidomain conductivity parameters (summarized by Roth [6]). On the other hand, the conductivity of blood, which is important in these simulations, is generally accepted to have a reasonably small variability at frequencies of up to $1 \mathrm{MHz}$ [7].

Roth [6] has presented a "recipe" for determining relative bidomain conductivities given nominal properties of the cardiac tissue. However, this recipe does not include the conductivity of blood, which is perhaps not surprising as many simulations using the bidomain model are primarily interested in the electrical stimulation of cardiac tissue [8]-[10]. On the other hand, simulations of defibrillation [11], [12] require blood conductivity values (as well as conductivities of other regions within the thorax).

This paper will, first, present a summary of the effect of the existing bidomain conductivity parameters on the epicardial potential distribution. Then, by normalizing the conductivities with respect to the extracellular conductivity in the longitudinal direction, it will use the recipe of Roth [6] to study the relative effect of blood conductivity on the epicardial potential distribution. Effects of other parameters, such as the ratio of longitudinal to transverse intracellular conductivity and anisotropy ratio, are also considered.

\section{METHODS}

\section{A. Model Description}

The model to be used in this paper has been described previously [3] and only a brief outline will be given. The cardiac tissue is represented by a block of tissue, infinite in the $x$ and $y$ coordinate directions and of unit thickness in the $z$ direction with the epicardium represented by the $x-y$ plane. The endocardium is, therefore, represented by the plane at $z=1$, in contact with a volume of blood extending to infinity in the positive $z$ direction.

The tissue is approximated by the bidomain model [4], [13], [14] for which the governing equation for the extracellular potential, $\phi_{e}$, can be shown to be

$$
\nabla \cdot\left(\mathbf{M}_{i}+\mathbf{M}_{e}\right) \nabla \phi_{e}=-\nabla \cdot \mathbf{M}_{i} \nabla \phi_{m}
$$

where $\phi_{m}=\phi_{i}-\phi_{e}$ is the transmembrane potential and $\phi_{i}$ is the intracellular potential. The tensors $\mathbf{M}_{e}$ and $\mathbf{M}_{i}$ reflect the anisotropic conductivity, as well as fiber rotation, within the cardiac tissue [3]. Anisotropic conductivity is given in terms of the intracellular and extracellular longitudinal and transverse 
conductivities $\sigma_{l}^{i}, \sigma_{t}^{i}, \sigma_{l}^{e}$, and $\sigma_{t}^{e}$. Also, the potential distribution in the blood, $\phi_{b}$, is governed by Laplace's equation

$$
\nabla^{2} \phi_{b}=0 .
$$

The accompanying boundary conditions are that the potentials tend to zero at large distances from the origin, that is, as $x \rightarrow$ $\pm \infty$ and $y \rightarrow \pm \infty, \phi_{e}=\phi_{b}=0$. Since the epicardium is assumed insulated, it follows that:

$$
\text { at } z=0 ; \quad \frac{\partial \phi_{e}}{\partial z}=0 .
$$

Further, at the interface between the tissue and the blood, there is continuity of potential and current, i.e.,

$$
\text { at } z=1 ; \quad \phi_{e}=\phi_{b} \text { and } \sigma_{b} \frac{\partial \phi_{b}}{\partial z}=\sigma_{t}^{e} \frac{\partial \phi_{e}}{\partial z}
$$

where $\sigma_{b}$ is the conductivity of blood. Finally, since the blood mass is assumed infinite in the positive $z$ direction, $\phi_{b}=0$ as $z \rightarrow \infty$.

Equation (1) is a Poisson equation for the electric potential in the extracellular space with the source term being the generalized Laplacian of the transmembrane potential distribution. Since it is assumed that there is a difference in plateau potentials between the normal and ischemic tissue, this term will be nonzero, thus creating potential distributions which are observed as ST depression or ST elevation on the body surface.

The fibers in the tissue will be assumed to rotate through an angle of $120^{\circ}$ (chosen for consistency with our previous model [3]). If the fibers on the epicardium are aligned along the positive $x$ axis, then the longitudinal direction at any depth $z$, with respect to the positive $x$ axis is given by

$$
g(z)=\frac{2 \pi}{3} z .
$$

As mentioned above, this rotation is taken into account in the conductivity tensors.

It will also be assumed that the region of subendocardial ischemia occupies a square finite region in the $x$ and $y$ directions, centered on the $z$ axis. In the $z$ direction, the region is bounded by the endocardium, but does not extend to the epicardium.

A technique for solving the governing equation [Equation (1)] has also been described previously [3] and so will only be described in outline here. A two-dimensional (2-D) Fourier transform in the $x$ and $y$ directions is applied to equation (1), resulting in an ordinary differential equation in the $z$ direction. This equation is solved for the Fourier transform of the potential in the extracellular space using a one-dimensional finite difference method with nodes clustered near the ischemic border. The resulting Fourier transforms are inverted to recover the extracellular potentials using a 2-D fast Fourier transform. Inversion in this fashion slightly changes the physics of the problem. Instead of dealing with a domain which is infinite in the $x$ and $y$ directions, the domain is now of finite extent and the boundary conditions are of an insulating type. In order to minimize the difference between these two situations, the computational domain is made large enough to allow the boundary potentials to approach zero.

\section{B. Modeling Parameters}

It will be assumed that the block of tissue modeled is $1 \mathrm{~cm}$ thick and $16 \mathrm{~cm}$ in each of the $x$ and $y$ directions. The region of ischemia is a square of side $4 \mathrm{~cm}$ centered on the origin and the parameters governing the width of the ischemic border are all $0.01 \mathrm{~cm}$ (see [3]), which reflects a narrow ischemic boundary. Finally, the difference between plateau potentials in normal and ischemic tissue, $\Delta \phi_{p}$, was set at $-30 \mathrm{mV}$.

The main aspect of this paper is to demonstrate the difference in epicardial surface potential distributions arising from the different conductivity values available in the literature. Some of the most frequently quoted values are given in Table I. As mentioned by Roth [6], there is a significant variation in each of these parameters, up to a factor of three, and not always in the same direction, from experiment to experiment. In contrast to this, the conductivity of blood exhibits a much smaller variation and is generally accepted to be about $0.0067 \mathrm{~S} / \mathrm{cm}$. Three sets of simulations based on these parameters are presented in Section III.

Roth [6] also mentions the auxiliary parameters $\lambda_{l}, \lambda_{t}$ (length constants of the tissue in the longitudinal and transverse directions, respectively), $\alpha$ (ratio of intracellular and extracellular conductivities) and $\epsilon$ (one minus the anisotropy ratio in the extracellular and intracellular spaces) (see [6] for the exact definitions). Values for these parameters are also included in Table I. Based on considerable experimental evidence, Roth [6] suggests the following nominal values for these parameters: $\lambda_{l} / \lambda_{t}=2.5$, $\alpha=1.0$, and $\epsilon=0.75$. Using these values, the following dimensionless bidomain conductivities (normalized with respect to $\left.\sigma_{l}^{i}\right)$ are obtained: $\left(\sigma_{l}^{i}\right)^{\prime}=\sigma_{l}^{i} / \sigma_{l}^{i}=1.0,\left(\sigma_{t}^{i}\right)^{\prime}=\sigma_{t}^{i} / \sigma_{l}^{i}=0.1$, $\left(\sigma_{l}^{e}\right)^{\prime}=\sigma_{l}^{e} / \sigma_{l}^{i}=1.0$, and $\left(\sigma_{t}^{e}\right)^{\prime}=\sigma_{t}^{e} / \sigma_{l}^{i}=0.4$.

The next step is to consider normalizing the conductivity of blood. The natural approach would be to normalize with respect to $\sigma_{l}^{i}$, as suggested by Roth [6]. However, given that the conductivity of blood is incorporated in the model through the boundary condition (4), which also contains $\sigma_{t}^{e}$, it would be more appropriate to normalize all the conductivities with respect to one of the extracellular conductivities. In view of the nominal parameters chosen by Roth [6], it was decided to normalize the conductivities with respect to $\sigma_{l}^{e}$, thus preserving the straightforward nature of Roth's dimensionless representation and giving

$$
\begin{aligned}
& \left(\sigma_{l}^{i}\right)^{\prime}=\sigma_{l}^{i} / \sigma_{l}^{e}=\alpha \\
& \left(\sigma_{t}^{i}\right)^{\prime}=\sigma_{t}^{i} / \sigma_{l}^{e}=\left(\frac{\lambda_{t}}{\lambda_{l}}\right)^{2} \frac{\alpha(1+\alpha(1-\epsilon))}{1+\alpha} \\
& \left(\sigma_{l}^{e}\right)^{\prime}=\sigma_{l}^{e} / \sigma_{l}^{e}=1.0 \\
& \left(\sigma_{t}^{e}\right)^{\prime}=\sigma_{t}^{e} / \sigma_{l}^{e}=\left(\frac{\lambda_{t}}{\lambda_{l}}\right)^{2} \frac{1+\alpha(1-\epsilon)}{(1+\alpha)(1-\epsilon)} .
\end{aligned}
$$

Using Roth's nominal parameters yields the same values for the dimensionless conductivities as with the original normalization.

In a similar fashion, define the dimensionless blood conductivity as

$$
\sigma_{b}^{\prime}=\frac{\sigma_{b}}{\sigma_{l}^{e}}
$$


TABLE I

VALUes of Bidomain Tissue CONDUCTIVITIES From THE INDiCATED SOURCES (IN S/cm), THEIR CORRESPONDING DIMENSIONLESS VALUES AND, VALUES OF THE AUXILIARY DiMENSIONLESS PARAMETERS

\begin{tabular}{c|c|c|c}
\hline & Clerc [5] & Roberts et al. [15] & Roberts and Scher [16] \\
\hline$\sigma_{l}^{i}$ & 0.00174 & 0.0028 & 0.0034 \\
$\sigma_{t}^{i}$ & 0.000193 & 0.00026 & 0.0006 \\
$\sigma_{l}^{e}$ & 0.00625 & 0.0022 & 0.0012 \\
$\sigma_{t}^{e}$ & 0.00236 & 0.0013 & 0.0008 \\
$\sigma_{b}$ & 0.0067 & 0.0067 & 0.0067 \\
\hline$\left(\sigma_{l}^{i}\right)^{\prime}(=\alpha)$ & 0.2784 & 1.2727 & 2.8333 \\
$\left(\sigma_{t}^{i}\right)^{\prime}$ & 0.0309 & 0.1182 & 0.5000 \\
$\left(\sigma_{l}^{e}\right)^{\prime}$ & 1.0000 & 1.0000 & 1.0000 \\
$\left(\sigma_{t}^{e}\right)^{\prime}$ & 0.3776 & 0.5909 & 0.6667 \\
\hline Anisotropy Ratio & 3.4043 & 6.3636 & 3.7778 \\
$\epsilon$ & 0.7063 & 0.8429 & 0.7353 \\
$\lambda_{l} / \lambda_{t}$ & 2.7621 & 2.3846 & 1.6084 \\
$\sigma_{b}^{\prime}$ & 1.072 & 3.0455 & 5.5833 \\
\hline
\end{tabular}

Values for this quantity are given in Table I for the three available data sets of conductivities. As can be seen from the table, the value of $\sigma_{b}^{\prime}$ ranges from 1 up to about 6 .

\section{RESULTS}

Figs. 1-3 show the epicardial surface potential distributions for varying wall thickness ischemia using the three data sets given in Table I. Each figure shows the epicardial potential distribution when the inner 10\% [panel (a)], 40\% [panel (b)], 70\% [panel (c)], and 90\% [panel (d)] of the wall is ischemic.

First, consider Fig. 1 obtained using the conductivity values determined by Clerc [5]. At 10\% ischemia, the potential distribution could be described as a valley (ST depression) with almost circular cross section, but at $40 \%$ ischemia, the valley has three distinct minima and there is an encroaching zero line extending from the positive and negative $x$ directions. The depths of these valleys are shallower than those at $10 \%$ ischemia. At $70 \%$ ischemia there is a breakthrough of positive potential (ST elevation) on the epicardial surface, directly above the positive and negative $y$ borders of the ischemic zone, which extend to the sides of the region, and the valleys have separated into three distinct regions. At this point, the depths of the valleys have again increased and there is the first indication of the large potential gradients above the border of the ischemic region. At $90 \%$ ischemia, most of the potential above the ischemic region is positive, except for a small region in the center. The valleys to either side of the ischemic region are again deeper than previously. With full thickness ischemia (not shown here, see [3]), there are high potential gradients around the ischemic border, the potential is always positive above the ischemic region, being higher toward the edges. Finally, both the positive and negative potentials are more extreme than in any previous situation.

Now, consider the same sequence of plots based on the data of Roberts et al. [15] (Fig. 2). The first thing to observe is that the epicardial potentials have greater magnitudes than those generated from the data of Clerc and the pattern of increasing/decreasing of potentials with degree of ischemia is similar. With this in mind, there is not a great difference between the epicardial potential distributions up to about 50\% ischemia (not shown), except that the potential gradients are significantly higher. However, at $70 \%$ the ST elevation above the ischemic border does not extend to the boundary of the region and at $90 \%$ ischemia, the region of positive potential above the ischemic region is isolated by negative potential on all sides. Finally, at full thickness ischemia (not shown), the patterns are again similar to the patterns from Clerc's data, except that the magnitudes are more extreme, resulting in larger potential gradients.

A different situation arises when the data of Roberts and Scher [16] are used (Fig. 3). First, observe that in general, the potentials are much more extreme than in the two previous cases and the negative potentials have smaller variations in magnitude as the degree of ischemia increases. Another observation is that the three distinct valleys that appear in the previous two cases do not appear for this data set. At 70\% ischemia there is evidence of large potential gradients, but no suggestion of the ST elevation as seen in the previous situations. Even at $90 \%$ ischemia, there is no significant positive potential observed; however, there are large potential gradients at the $y$ direction extremes of the ischemic boundary. The potential distribution at full thickness ischemia is not significantly different from those distributions obtained using the first two data sets described previously, except that the magnitudes of the potentials are greater.

Fig. 4 shows the epicardial potential distribution at $70 \%$ subendocardial ischemia obtained for various values of the parameter $\alpha$ with $\sigma_{b}^{\prime}=1$ and $\epsilon=0.75$. A value of $\alpha=0.25$ [Fig. 4(a)] yields a distribution similar to that obtained using the data set of Clerc [5] [Fig. 1 (c)]. Increasing $\alpha$ to 1 Fig. 4(b)] again yields a distribution similar to that obtained using the data set of Clerc [5] (Fig. 1), but has magnitudes more of the order of the potential obtained from the data of Roberts et al. . As $\alpha$ is increased to 2 Fig. 4(c)] the distribution becomes similar to that obtained using the data set of Roberts et al. [15] (although the positive regions are smaller). Finally, with $\alpha=3$ Fig. 4(d)] the distributions obtained approach that obtained from the data of Roberts and Scher [16], although the magnitudes are somewhat smaller.

In order to assess the effect of the ratio of blood to tissue conductivity, simulations were performed with the dimensionless tissue conductivity values given in Section II and values of $\sigma_{b}^{\prime}=1,2$, and 3 . For the purposes of illustration, epicardial potentials are only shown for $70 \%$ ischemia as this showed the greatest degree of variation with the original data sets. The resulting potential distributions are shown in Fig. 5. With $\sigma_{b}^{\prime}=1$ [Fig. 5(a)], there is a great similarity to distributions obtained from the data of Clerc (Fig. 1), except that the magnitudes of the potentials are greater (more of the order of the potentials obtained from the data of Roberts et al.). When $\sigma_{b}^{\prime}=2$ [Fig. 5(b)], there are small regions of ST elevation near two corners of the ischemic region. This is similar to the distribution from the data 


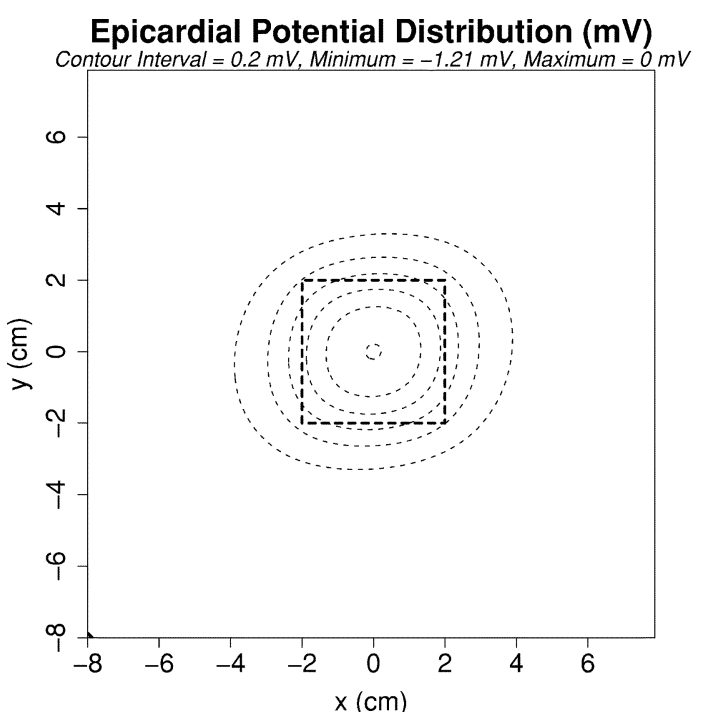

(a)

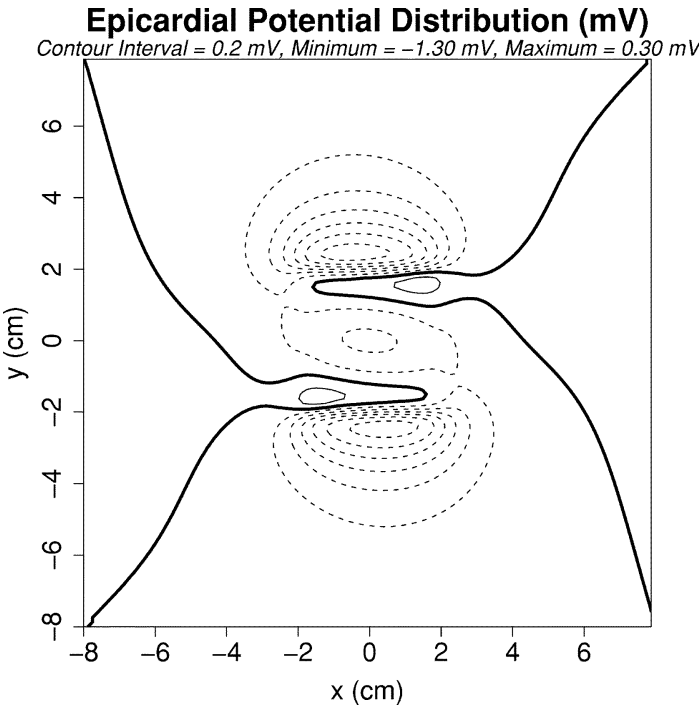

(c)

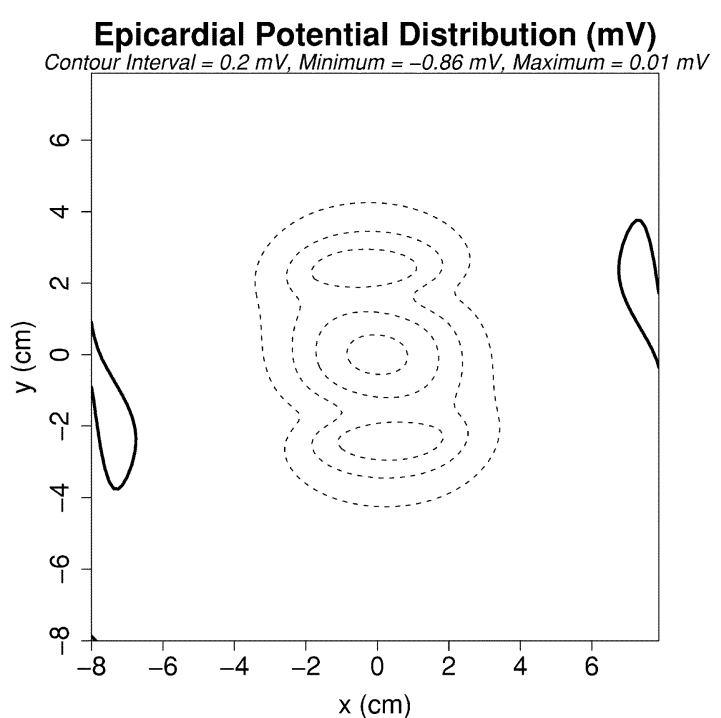

(b)

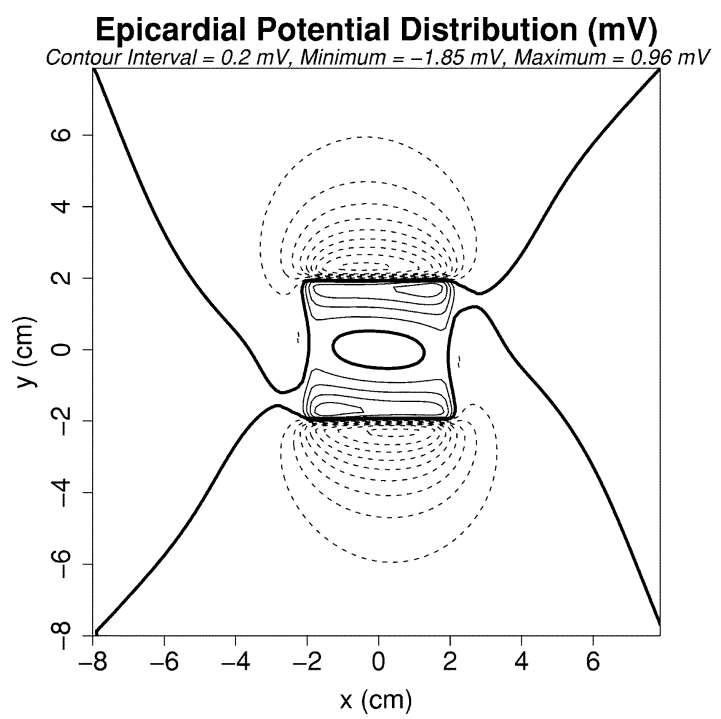

(d)

Fig. 1. Epicardial surface potential distributions during the transition to full thickness ischemia using the conductivity data of Clerc [5]. Distributions are shown for subendocardial ischemia at (a) $10 \%$, (b) $40 \%$, (c) $70 \%$, and (d) $90 \%$. Each panel shows the maximum and minimum potential value, with a contour interval of $0.2 \mathrm{mV}$. The solid lines represent positive potential, the broken lines, negative potential and the thick solid line is the zero of potential. The thick dashed line in (a) represents the projection of the ischemic region onto the epicardium (this region is common to all subsequent contour plots).

of Roberts et al., except that these positive regions are considerably smaller. Finally, with $\sigma_{b}^{\prime}=3$ [Fig. 5(c)], a potential distribution similar to that obtained from the data of Roberts and Scher is observed. The similarity lies in the fact that the potential is generally negative everywhere and there are large potential gradients near the ischemic boundary; however, this simulation has several minima in the potential distribution, as opposed to that shown in Fig. 3. It is interesting to note that increasing $\sigma_{b}^{\prime}$ above three does not greatly affect the resulting potential distributions in either shape or magnitude.

\section{DISCUSSION}

This paper has investigated the effect electrical conductivity values have on ST segment epicardial potential distributions induced by subendocardial ischemia. Three sets of measured tissue conductivity values were used to obtain the epicardial po- tentials and then a normalized set of conductivities were used to consider the relative effect of blood conductivity.

The effect of the three measured sets of tissue conductivity on epicardial potential distributions is shown in Figs. 1-3. The main observations are the changes in morphology and potential magnitude between the data sets. All simulated potentials are smaller in magnitude than those encountered in the experiments of Li et al. [17]. It should be noted that the data from Li et al. were derived from true ischemia in either the territory of the left anterior descending (LAD) or circumflex (CX) coronary arteries over the epicardium of an in vivo heart. The heart includes the septum and right ventricular blood volume, not included in this model.

The conclusions from the experimental data were that the position of the ST depression maximizes over the left ventricular front wall, which is the boundary between the LAD and CX artery territories. Experiments with progression of ischemia can not accurately say how much myocardium is ischemic when ST 


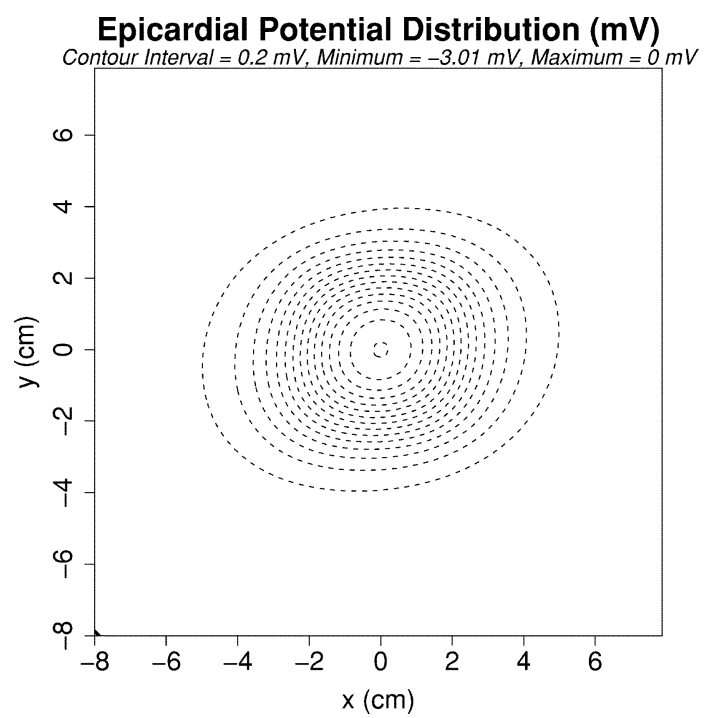

(a)

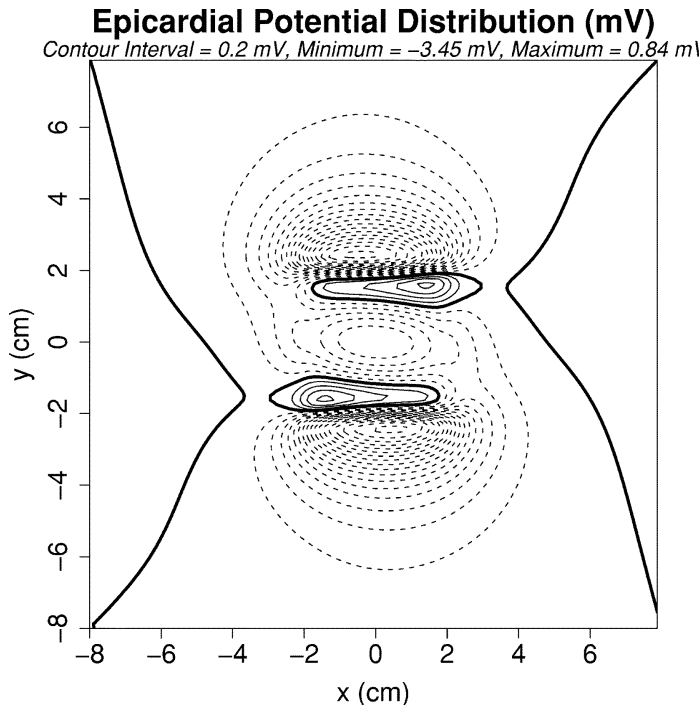

(c)

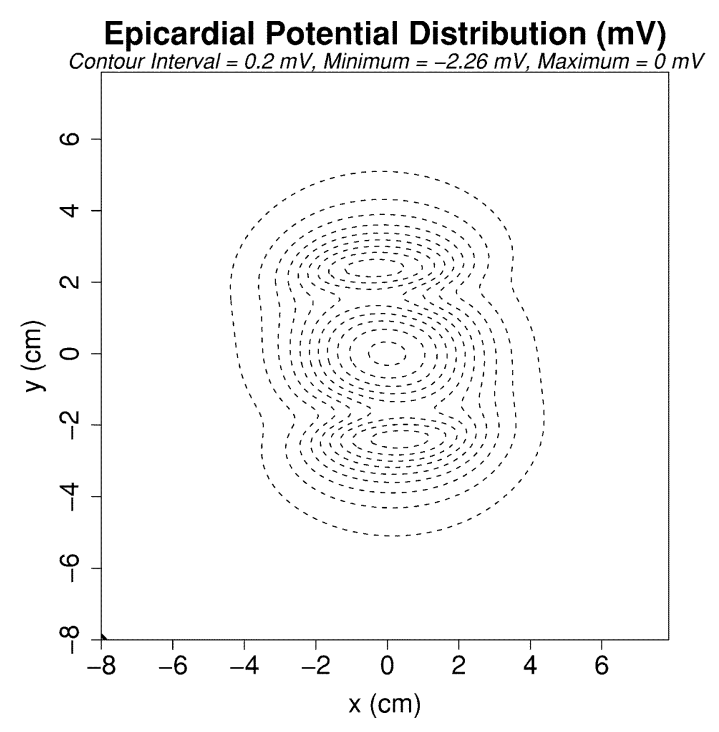

(b)

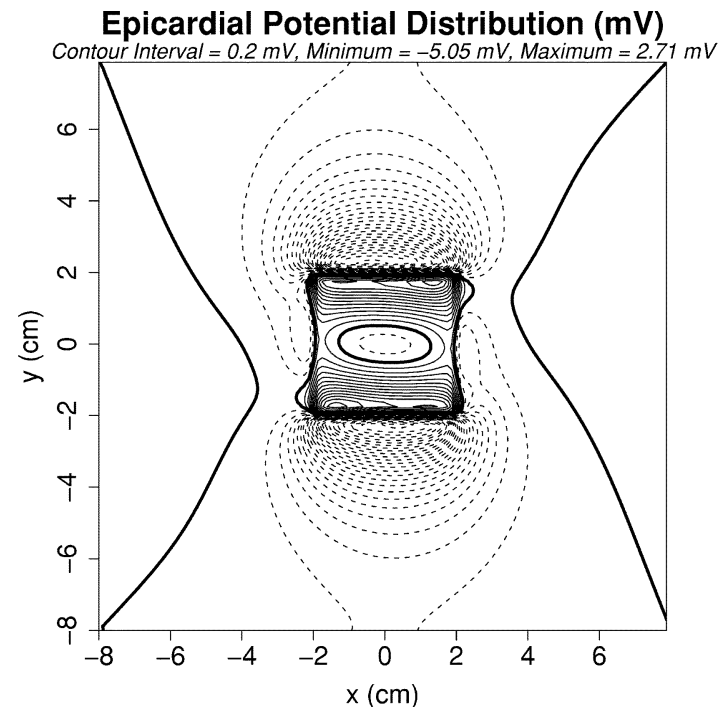

(d)

Fig. 2. Epicardial surface potential distributions during the transition to full thickness ischemia using the conductivity data of Roberts et al. [15]. The format of the figure is the same as Fig. 1.

elevation develops, but it is believed to be nearly $100 \%$. The ST depression is increased as the degree of ischemia is increased and when approaching full thickness ischemia, ST elevation moves from the boundary of the ischemic region to be situated above its center.

A further difference between the model and the experimental data is in the choice of the reference. Although this does not alter the pattern, the degree of positivity or negativity is obviously affected by the reference electrode chosen. In addition, the model uses a fixed potential difference between transmembrane potentials for ischemic and normal tissue, whereas, in experimental work, this driving potential is dependent on the time after the onset of ischemia.

An additional factor in experimental ischemia is that it is not absolute. The technique used is to reduce flow by a snare and increase work by pacing, resulting in graduated ischemia. Additionally, the electrophysiologic properties of the ischemic boundary are not accurately known.
As mentioned previously [3], this model (using the conductivity data of Clerc [5]) predicted a relationship between the regions of subendocardial ischemia and the position of ST depression over the epicardium, an observation in contrast to published experimental data [18], [17]. It was also mentioned that the magnitudes of the simulated potential data were smaller than those observed experimentally [17]. However, the model did predict the high potential gradients near the border of the ischemic region, and that ST depression increased before the occurrence of ST elevation and continued to increase as ST elevation increased.

Using the conductivity data of Roberts et al. [15], results in simulated potential values which are closer in magnitude to the experimentally measured values (Fig. 2). However, the model still suggests that the epicardial ST depression pattern localizes the subendocardial ischemia. The model, with this conductivity data, still predicts the ST elevation and the high potential gradients near the ischemic border. Hence, in one sense, using this set of conductivity data brings the model closer to the experimental data. 


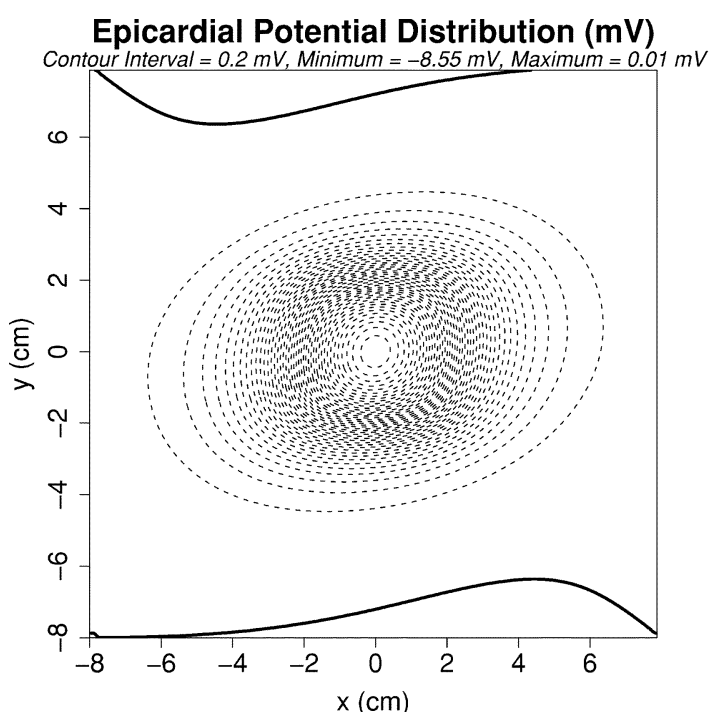

(a)

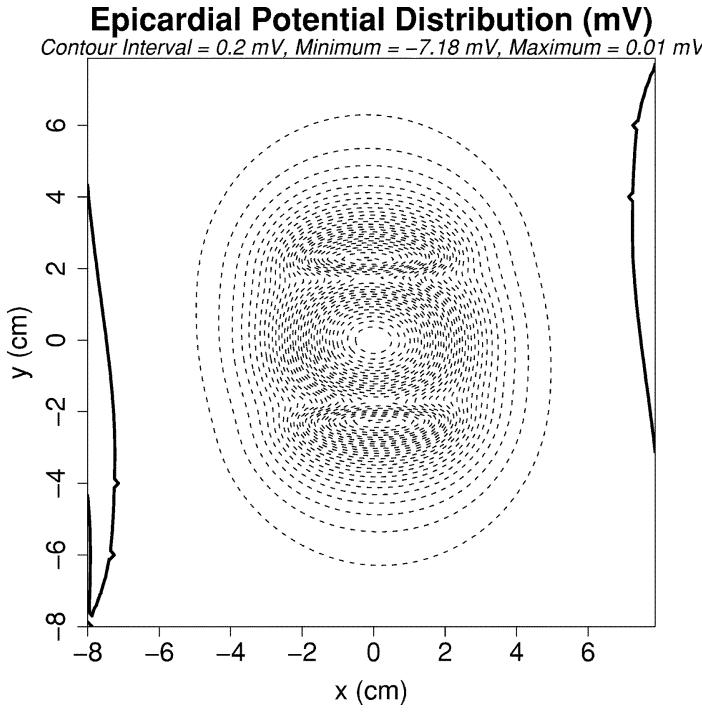

(c)

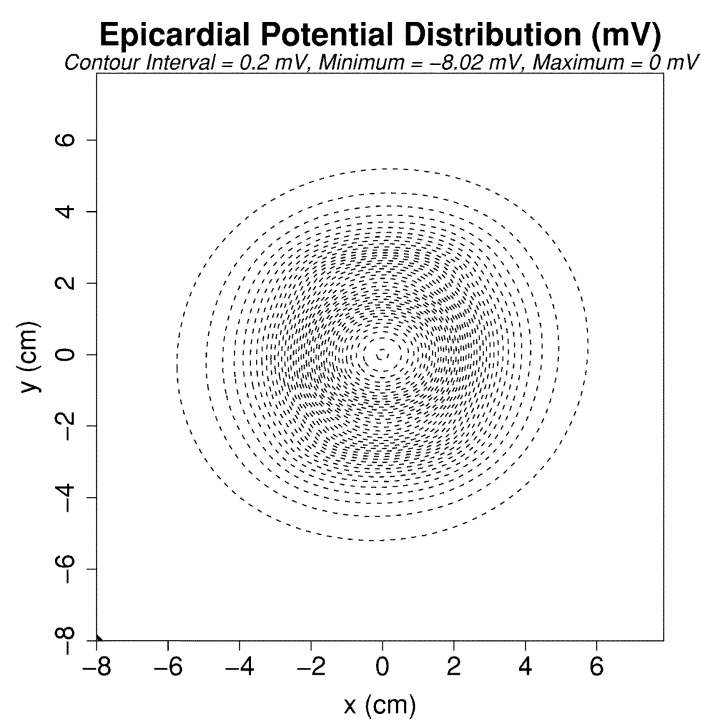

(b)

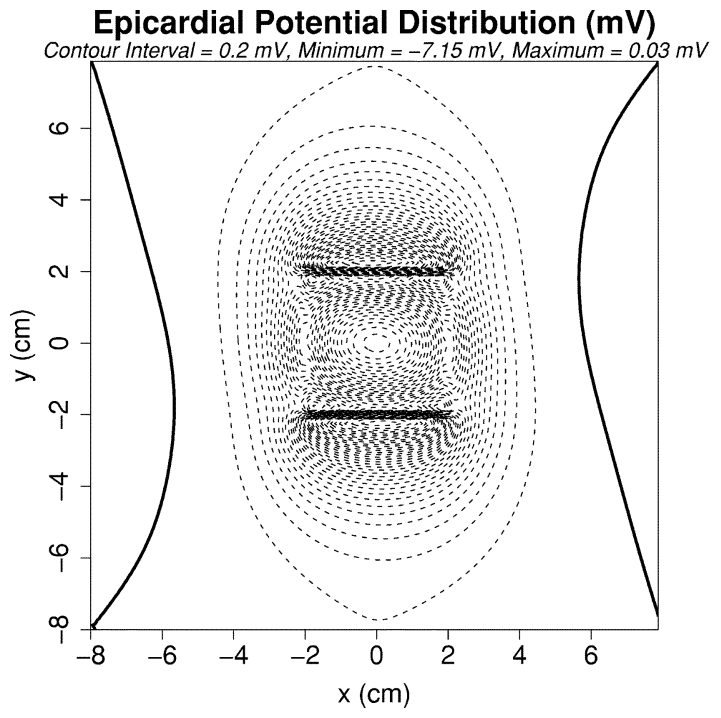

(d)

Fig. 3. Epicardial surface potential distributions during the transition to full thickness ischemia using the conductivity data of Roberts and Scher [16]. The format of the figure is the same as Fig. 1.

Finally, using the conductivity data of Roberts and Scher [16] results in a completely different set of epicardial potential distributions. To begin with, the magnitudes of the potential are closer to the experimental data than either of the previous data sets. However, there is no ST elevation observed over the ischemic region until the ischemia is nearly full thickness, which would fit with the experimental evidence [17]. On the other hand, there is no increase in ST depression with increasing ischemia as suggested by the experimental data. The model is still consistent in that it localizes the region of ischemia and there are high potential gradients near the ischemic border.

The transition in results from Figs. 1 to 2 to 3 is perhaps somewhat unexpected. First, going from Fig. 1 to 2 yields potential distributions of similar morphology but with increasing magnitudes. This might be expected as there is an increase in intracellular conductivities and a decrease in extracellular conductivities between the data sets of Clerc [5] and Roberts et al. [15]. However, continuing with this idea, the results in Fig. 3 would not be expected. Again there is an increase in the intracellular conductivities and a decrease in the extracellular conductivities, but the resulting potential distributions are completely different. It might be expected that increases in ST depression for this data would be matched with an increase in ST elevation and a similar distribution to the first two data sets.

On the other hand, considering just the fact that the three data sets yield different magnitudes of potential, a possible explanation for this is the increasing $\alpha$ values (Table I) for the three data sets. The values of $\alpha$ increase as 0.2784 (Clerc), 1.2727 (Roberts et al.), and 2.8333 (Roberts and Scher), which matches the trend of increasing potential magnitudes. To test this hypothesis, simulations were performed with $\alpha$ values ranging from 0.25 up to 3 (keeping other values constant) (Fig. 4). The results tended to match the general trend in shapes and magnitudes of the epicardial distributions for the three different sets of measured conductivity parameters considered. Hence, varying this one parameter can explain some trends in the data.

It has been mentioned previously [3] that a possible reason for the smaller than expected potential magnitudes in the model was 
Epicardial Potential Distribution ( $\mathrm{mV})$

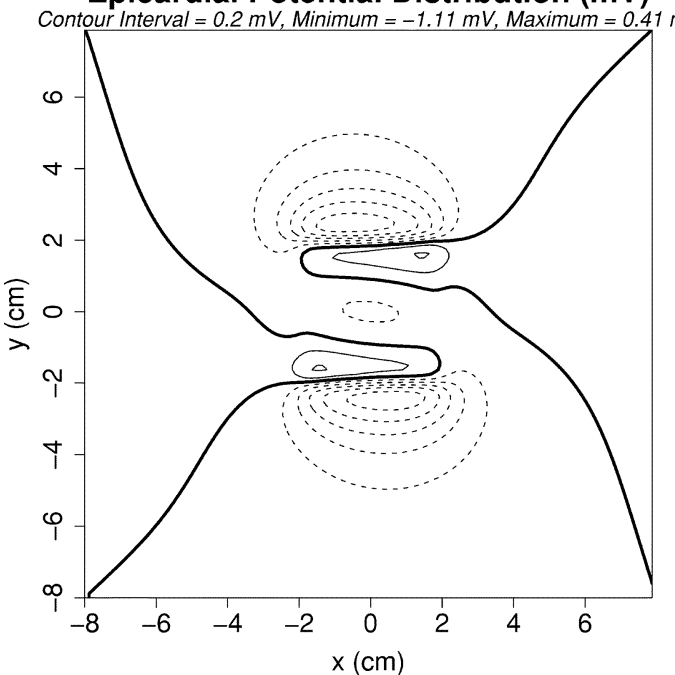

(a)

Epicardial Potential Distribution (mV)

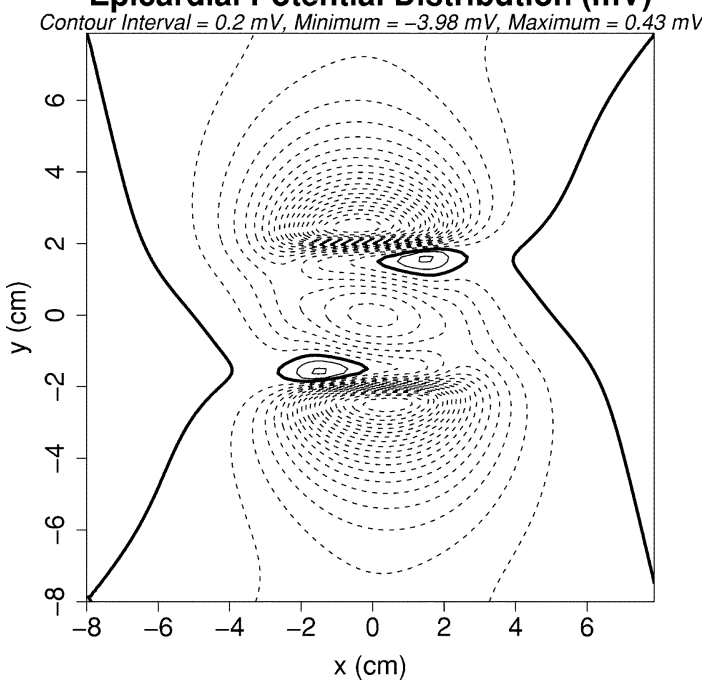

(c)
Epicardial Potential Distribution (mV)

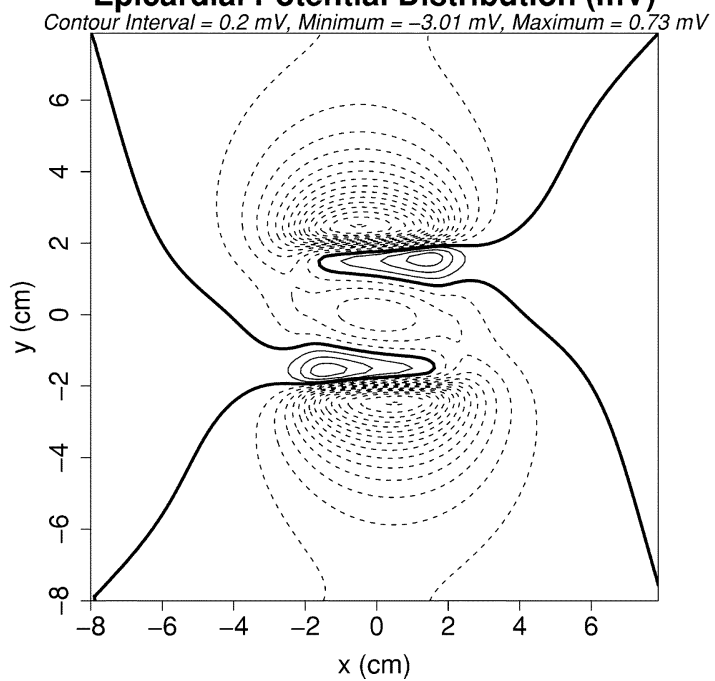

(b)

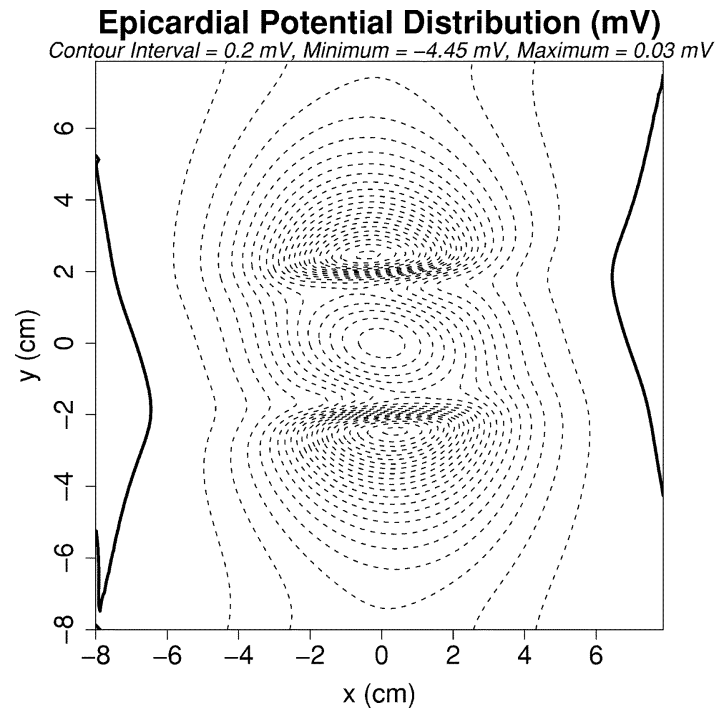

(d)

Fig. 4. Epicardial potential distributions at 70\% subendocardial ischemia with $\sigma_{b}^{\prime}=1$ and $\epsilon=0.75$. The panels correspond to different values of $\alpha$ : (a) $\alpha=0.25$, (b) $\alpha=1$, (c) $\alpha=2$, and (d) $\alpha=3$. The format of each panel is the same as in Fig. 1.

the presence of the infinite blood mass. To investigate this hypothesis, simulations were performed with various values of $\sigma_{b}^{\prime}$ (Fig. 5). Since it is assumed that the blood conductivity is constant (due to its generally accepted value), changes in $\sigma_{b}^{\prime}$ represent changes in the tissue conductivity values (all other parameters remaining constant). From Table I, it can be seen that increasing $\sigma_{b}^{\prime}$ also corresponds to increasing potential magnitudes. The simulations revealed the same change in potential patterns as with the variation in tabulated tissue conductivity parameters, but, surprisingly, the magnitudes of the potentials were reasonably similar. Hence, varying $\sigma_{b}^{\prime}$ can also explain the change in potential patterns, but not the change in magnitude. Therefore, the relative conductivities of blood and tissue are not considered to be important as changes in the value of $\alpha$ can account for changes in morphology and magnitude of the potential distribution.

Now consider the effects of $\epsilon$ and the ratio $\lambda_{l} / \lambda_{t}$. It can be seen that for a fixed value of $\alpha$, increasing $\epsilon$ and decreasing $\lambda_{l} / \lambda_{t}$ both increase the dimensionless transverse conductivities $\left(\sigma_{t}^{i}\right)^{\prime}$ and $\left(\sigma_{t}^{e}\right)^{\prime}$. It turns out that increasing these conductivities allows ST elevation to occur at lesser degrees of subendocardial ischemia, thereby resulting in earlier ST elevation over the ischemic region. Again, changing these conductivity values has little effect on the magnitude of the potentials. On the other hand, decreasing these conductivities tends to result in ST depression occurring over the entire surface with sites of ST elevation occurring only toward full thickness ischemia.

From this discussion, it can be concluded that there is a complex interplay between the parameters in this model and the resulting epicardial potential distributions.

\section{CONSLUSION}

This paper has used a previously proposed bidomain model of cardiac tissue to study the effects of tissue and blood conductivity on the behavior of ST depression and elevation during subendocardial ischemia. The model can be described in terms 
Epicardial Potential Distribution ( $\mathrm{mV}$ )

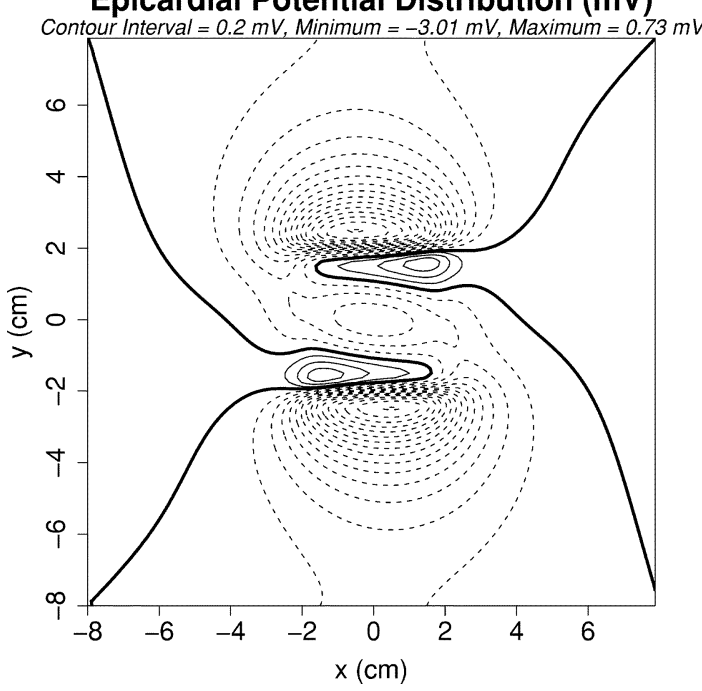

(a)

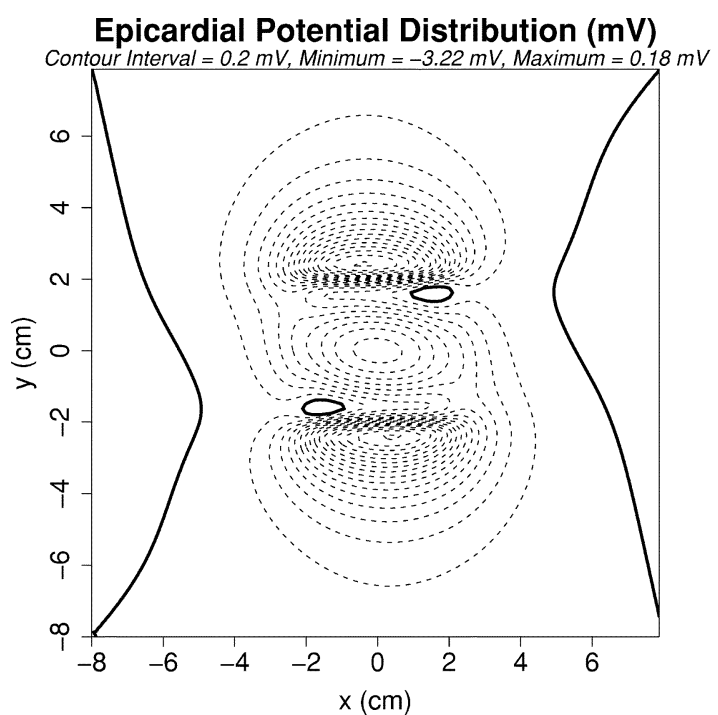

(b)

Epicardial Potential Distribution (mV)

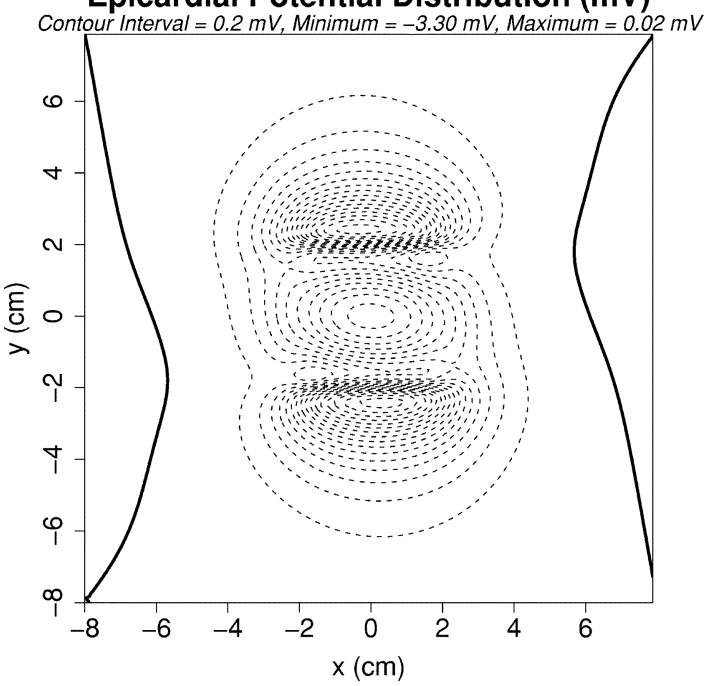

(c)

Fig. 5. Epicardial potential distributions at $70 \%$ subendocardial ischemia with $\alpha=1$ and $\epsilon=0.75$, with an anisotropic ratio of 4 . The panels correspond to different values of $\sigma_{b}^{\prime}$ : (a) $\sigma_{b}^{\prime}=1$, (b) $\sigma_{b}^{\prime}=2$, and (c) $\sigma_{b}^{\prime}=3$. The format of each panel is the same as in Fig. 1.

of the four parameters $\alpha, \epsilon, \lambda_{l} / \lambda_{t}$, and $\sigma_{b}^{\prime}$, which have been varied both in accordance with experimental values and artificially. Note that a slight variation in the definitions of the dimensionless conductivities has been introduced to that used previously [6].

Based on a comparison of the morphology and magnitudes of the simulated and measured epicardial surface potential distributions, it could be argued that the conductivity values proposed by Roberts et al. [15] should be used for the purposes of simulation with realistic conductivity values. On the other hand, for simulations based on the dimensionless representations of Roth [6] with modifications suggested here, these values should be augmented with a dimensionless blood conductivity parameter $\sigma_{b}^{\prime}$ of about one, or slightly greater.

However, in terms of a comparison with measured data, there are still shortcomings in the model regarding the actual magnitude of the potentials, as well as its ability to localize subendocardial ischemia. Clearly, these two anomalies cannot be ex- plained with the simple geometric character of the model presented. A more geometrically realistic model is required to answer all the questions posed by this complex situation.

Accurate values for conductivity parameters are necessary and may need to reflect the individual experiment. A further assumption which needs to be tested is that conductivity values for ischemic muscle are the same as for normal muscle. These must be determined from an experimental model.

\section{REFERENCES}

[1] C. C. Wolferth, S. Bettet, M. M. Livezey, and F. Murphy, "Negative displacement of the RS-T segment in the electrocardiogram and its relationships to positive displacement: An experimental study," Amer. Heart J., vol. 29 , pp. 220-244, 1945

[2] H. Toyoshima, A. Ekmekci, E. Flamm, Y. Mizuno, T. Nagaya, R. Nakayama, K. Yamada, and M. Prinzmetal, "Angina pectoris VII. The nature of ST depression in acute myocardial ischemia," American J. Cardiol., vol. 13, pp. 498-509, 1964.

[3] P. R. Johnston, D. Kilpatrick, and C. Y. Li, "The importance of anisotropy in modeling ST segment shift in subendocardial ischemia," IEEE Trans. Biomed. Eng., vol. 48, pp. 1366-1376, Dec. 2001. 
[4] O. H. Schmitt, "Biological information processing using the concept of interpenetrating domains," in Information Processing in the Nervous System, K. N. Leibovic, Ed. New York: Springer-Verlag, 1969, ch. 18, pp. $325-331$.

[5] L. Clerc, "Directional differences of impulse spread in trabecular muscle from mammalian heart," J. Physiol., vol. 255, pp. 335-346, 1976.

[6] B. J. Roth, "Electrical conductivity values used with the bidomain model of cardiac tissue," IEEE Trans. Biomed. Eng., vol. 44, pp. 326-328, Apr. 1997.

[7] K. R. Foster and H. P. Schwan, "Dielectric properties of tissue and biological materials: A critical review," Crit. Rev. Biomed. Eng., vol. 17, no. 1 , pp. 25-104, 1989.

[8] B. J. Roth, "A mathematical model of make and break electrical stimulation of cardiac tissue by a unipolar anode or cathode," IEEE Trans. Biomed. Eng., vol. 42, pp. 1174-1184, Dec. 1995.

[9] K. B. Skouibine, N. A. Trayanova, and P. K. Moore, "Anode/cathode make and break phenomena in a model of defibrillation," IEEE Trans. Biomed. Eng., vol. 46, pp. 769-777, July 1999.

[10] A. Cimponeriu, C. F. Starmer, and A. Bezerianos, "A theoretical analysis of acute ischemia and infarction using ECG reconstruction on a 2-D model of myocardium," IEEE Trans. Biomed. Eng., vol. 48, pp. 41-54, Jan. 2001.

[11] N. A. Trayanova, B. J. Roth, and L. J. Malden, "The response of a spherical heart to a uniform electric field: A bidomain analysis of cardiac stimulation," IEEE Trans. Biomed. Eng., vol. 40, pp. 899-908, Sept. 1993.

[12] Y. Wang, D. R. Haynor, and Y. Kim, "An investigation of the importance of myocardial anisotropy in finite-element modeling of the heart: Methodology and application to the estimation of defibrillation efficacy," IEEE Trans. Biomed. Eng., vol. 48, pp. 1377-1389, Dec. 2001.

[13] L. Tung, "A Bi-domain model for describing ischemic myocardial D-C potentials," Ph.D. dissertation, Massachusetts Inst. Technol., Cambridge, June 1978.

[14] W. T. Miller and D. B. Geselowitz, "Simulation studies of the electrocardiogram: I the normal heart," Circ. Res., vol. 43, pp. 301-315, 1978.

[15] D. E. Roberts, L. T. Hersh, and A. M. Scher, "Influence of cardiac fiber orientation on wavefront voltage, conduction velocity and tissue resistivity in the dog," Circ. Res., vol. 44, pp. 701-712, 1979.

[16] D. E. Roberts and A. M. Scher, "Effects of tissue anisotropy on extracellular potential fields in canine myocardium in situ," Circ. Res., vol. 50, pp. 342-351, 1982.

[17] D. Li, C. Y. Li, A. C. Yong, and D. Kilpatrick, "Source of electrocardiographic ST changes in subendocardial ischemia," Circ. Res., vol. 82, pp. 957-970, 1998
[18] R. A. Guyton, J. H. McClethan, G. E. Newman, and L. L. Michaelis, "Significance of subendocardial S-T segment elevation caused by coronary stenosis in the dog," Amer. J. Cardiol., vol. 40, pp. 373-380, 1977.

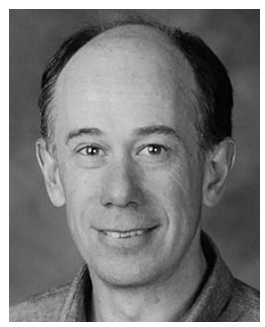

Peter R. Johnston was born in Hobart, Tasmania, Australia, in 1960. He received the Honors degree in mathematics from the University of Tasmania, Tasmania, Australia, in 1983, and the Ph.D. degree from the School of Chemical Engineering, the University of Queensland, Queensland, Australia, in 1987.

Thereafter, he worked in industry for three years before being employed as Senior Lecturer at the University of Tasmania, conjointly in the Departments of Medicine and Mathematics. He is now a Senior Lecturer in Mathematics at Griffith University, Brisbane, Queensland. His current areas of interest include the forward and inverse problems in electrocardiology, solution techniques for differential equations, and modeling blood flow using computational fluid dynamics.

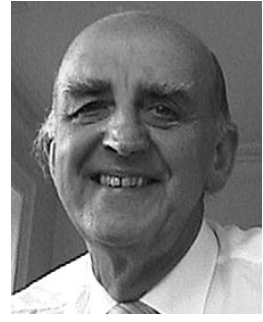

David Kilpatrick received the B.Sc. degree in physics and mathematics and the M.B.Ch.B. and M.D. degrees from the University of Otago, Dunedin, New Zealand, in 1967, 1972, and 1981, respectively. He also received the M.R.C.P. (UK) and F.R.A.C.P. degrees in 1979 and 1983, respectively.

$\mathrm{He}$ is an unemployed physicist currently working as a Professor of Medicine in the University of Tasmania, Tasmania, Australia. He was a commonwealth scholar at the Royal Postgraduate Medical School, Hammersmith Hospital, London, U.K., 1974-1979 and received further clinical training at Dalhousie, Nova Scotia, Nova Scotia, Canada, and the University of California at San Francisco before moving to Australia. His current interests include the origin of the electrocardiogram, coronary blood flow, and fiber-optic laser Doppler anemometry. Most of the time he works as a cardiologist. 